\title{
SMC X-3: the closest ultraluminous X-ray source powered by a neutron star with non-dipole magnetic field
}

\author{
S. S. Tsygankov ${ }^{1,2}$, V. Doroshenko ${ }^{3}$, A. A. Lutovinov ${ }^{2,4}$, A. A. Mushtukov ${ }^{5,6,2}$, and J. Poutanen ${ }^{1,7}$ \\ 1 Tuorla Observatory, Department of Physics and Astronomy, University of Turku, Väisäläntie 20, 21500 Piikkiö, Finland \\ e-mail: stsygankov@gmail.com \\ 2 Space Research Institute of the Russian Academy of Sciences, Profsoyuznaya Str. 84/32, 117997 Moscow, Russia \\ 3 Institut für Astronomie und Astrophysik, Universität Tübingen, Sand 1, 72076 Tübingen, Germany \\ ${ }_{5}^{4}$ Moscow Institute of Physics and Technology, Moscow region, Dolgoprudnyi, Russia \\ 5 Anton Pannekoek Institute, University of Amsterdam, Science Park 904, 1098 XH Amsterdam, The Netherlands \\ 6 Pulkovo Observatory of the Russian Academy of Sciences, 196140 Saint Petersburg, Russia \\ 7 Nordita, KTH Royal Institute of Technology and Stockholm University, Roslagstullsbacken 23, 10691 Stockholm, Sweden
}

Received 3 February 2017 / Accepted 16 May 2017

\begin{abstract}
Aims. The magnetic field of accreting neutron stars determines their overall behavior including the maximum possible luminosity Some models require an above-average magnetic field strength $\left(\gtrsim 10^{13} \mathrm{G}\right)$ in order to explain super-Eddington mass accretion rate in the recently discovered class of pulsating ultraluminous X-ray sources (ULX). The peak luminosity of SMC X-3 during its major outburst in 2016-2017 reached $\sim 2.5 \times 10^{39} \mathrm{erg} \mathrm{s}^{-1}$ comparable to that in ULXs thus making this source the nearest ULX-pulsar. Determination of the magnetic field of SMC X-3 is the main goal of this paper.

Methods. SMC X-3 belongs to the class of transient X-ray pulsars with Be optical companions, and exhibited a giant outburst in July 2016-March 2017. The source has been observed over the entire outburst with the Swift/XRT and Fermi/GBM telescopes, as well as the NUSTAR observatory. Collected data allowed us to estimate the magnetic field strength of the neutron star in SMC X-3 using several independent methods.

Results. Spin evolution of the source during and between the outbursts, and the luminosity of the transition to the so-called propeller regime in the range of $(0.3-7) \times 10^{35} \mathrm{erg} \mathrm{s}^{-1}$ imply a relatively weak dipole field of $(1-5) \times 10^{12} \mathrm{G}$. On the other hand, there is also evidence for a much stronger field in the immediate vicinity of the neutron star surface. In particular, transition from super- to sub-critical accretion regime associated with the cease of the accretion column and very high peak luminosity favor a field that is an order of magnitude stronger. This discrepancy makes SMCX-3 a good candidate for possessing significant non-dipolar components of the field, and an intermediate source between classical X-ray pulsars and accreting magnetars which may constitute an appreciable fraction of ULX population.
\end{abstract}

Key words. accretion, accretion disks - magnetic fields - X-rays: binaries - X-rays: individuals: SMC X-3

\section{Introduction}

Magnetic field of a neutron star (NS) defines observational properties for a broad range of systems. Strongly magnetized accreting NSs, or X-ray pulsars (XRPs), are among the most prominent NS systems and are being actively studied, particularly in connection with the possibility of super-Eddington accretion. The main source of information about the strength of magnetic fields in XRPs is associated with the so-called cyclotron scattering resonance absorption features (CSRF) observed in the energy spectra of some sources. Unfortunately, this method is restricted by a sensitivity and energy range of X-ray telescopes and relatively soft spectra of XRPs. This implies a very limited number of XRPs with known magnetic fields, all of which fall into a narrow range $B \sim(1-8) \times 10^{12} \mathrm{G}$ (see review by Walter et al. 2015) defined by aforementioned selection effects. At the same time magnetic fields of very bright XRPs (including the pulsating ultraluminous X-ray sources, ULXs) are expected to be $\gtrsim 10^{13} \mathrm{G}$ (Mushtukov et al. 2015a), and it would be very important to find sources with similar fields among the ordinary accreting XRPs.

Fortunately, besides the spectroscopy, pulsars timing properties can be used to independently estimate the magnetic field strength. Accretion torque affecting the NS depend on the magnetosphere size and, although model-dependent (see, e.g., Parfrey et al. 2016), can be used to estimate the field.

Detection of a centrifugal inhibition of accretion, known as the propeller effect (Illarionov \& Sunyaev 1975; Stella et al. 1986) can also be used to estimate the magnetosphere size and thus the magnetic field. The reliability of this approach has been recently demonstrated by Tsygankov et al. (2016a,b) and Lutovinov et al. (2017), who showed that the magnetic field values estimated from the propeller effect are in good agreement with measurements from independent methods (including the direct estimate based on the observed CSRF energies).

SMCX-3 was discovered with the $S A S-3$ observatory as a bright source in the Small Magellanic Cloud by Clark et al. (1978). The source was reported to have a luminosity of $7 \times$ $10^{37} \mathrm{erg} \mathrm{s}^{-1}$ in the $2-11 \mathrm{keV}$ energy band, and a relatively hard spectrum (with photon spectral index of $\simeq 1$ ). Despite the early proposed optical identification (van Paradijs et al. 1977; Crampton et al. 1978) the nature of the source remained uncertain for a long time. Only in 2004, Edge et al. (2004) using the Chandra data had shown that the $7.78 \mathrm{~s}$ pulsar found by Corbet et al. (2004) in the RXTE data can be identified 
with SMC X-3. Based on the precise X-ray position reported by Edge et al. (2004), Cowley \& Schmidtke (2004) studied the long term optical light curve of SMCX-3 and established its counterpart as a $09 \mathrm{e}$ star. A number of outbursts detected with the RXTE observatory in 1999-2009 allowed a periodicity to be found in the source activity, likely associated with the orbital motion with the period of $\simeq 45$ days (Corbet et al. 2004). Based on the long-term spin period evolution observed by the RXTE, Klus et al. (2014) estimated the expected magnetic field of the NS in SMC X-3 as $B \simeq 2.9 \times 10^{12} \mathrm{G}$. We note that in this case a CSRF in the source spectrum at the energy of $\sim 26 \mathrm{keV}$ should be observable (accounting for the gravitational redshift). Another estimate of the magnetic field strength $B \simeq 7.3 \times 10^{12} \mathrm{G}$ was obtained recently by Weng et al. (2017) based on the assumption that the source was spinning close to an equilibrium in the tail of the 2016-2017 outburst.

The current 2016-2017 outburst from SMCX-3 was detected with the MAXI monitor and was initially designated as a possible new source MAXI J0058-721 (Negoro et al. 2016). The follow-up observations with the Swift/XRT telescope allowed to establish, however, that the new transient is in fact a known source (Kennea et al. 2016b), and to perform a timing and spectroscopy of this object in soft X-rays (Kennea et al. 2016a). Moreover, follow-up observations with the NuSTAR observatory allowed to investigate the broadband spectrum of SMCX-3 for the first time. It was shown that the SMC X-3 spectrum in the 3$50 \mathrm{keV}$ energy band can be well described by an exponentially cutoff power law model with the photon index of $\Gamma \simeq 0.5$ and the folding energy of $E_{\text {fold }} \simeq 12 \mathrm{keV}$. Additionally a blackbody component with the temperature of $k T \simeq 1.78 \mathrm{keV}$ and an emission line from neutral iron with equivalent width of $70 \mathrm{eV}$ were observed in the spectrum (Pottschmidt et al. 2016). It is important to emphasize that no other obvious spectral features, including the CSRF, were reported (Pottschmidt et al. 2016).

Extremely high bolometric luminosity of the source $L_{\text {peak }} \sim$ $2.5 \times 10^{39} \mathrm{erg} \mathrm{s}^{-1}$, reached during this outburst, makes SMC X-3 unique among transient X-ray pulsars with Be optical companions (Be/XRPs). In fact, due to the high luminosity it can be formally attributed to the group of ULXs. Its proximity to Earth would make it the closest ULX in this case, and furthermore, the closest ULX-pulsar. In this work we present results of the monitoring program performed with the Swift/XRT and Fermi/GBM telescopes, as well as the $N u S T A R$ observatory during recent outburst in July 2016-March 2017. These data allowed us to estimate the magnetic field strength of the NS in the system using several independent methods and to conclude that the source likely has a non-dipole configuration of the magnetic field.

\section{Observations}

\subsection{Swift/XRT data}

The best facilities for long-term monitoring programs in a broad range of fluxes are currently provided by the Swift observatory (Gehrels et al. 2004). During the current 2016-2017 outburst regular observations of SMC X-3 were performed with the XRT telescope (Burrows et al. 2005) in the soft X-ray band (0.5$10 \mathrm{keV}$ ) providing both high sensitivity and flexibility. The data analyzed in this work were collected between MJD 57611 and MJD 57840.

The XRT telescope observed SMCX-3 in both windowed timing (WT; providing good temporal resolution) and photon counting (PC) modes. Final scientific products (spectrum in each

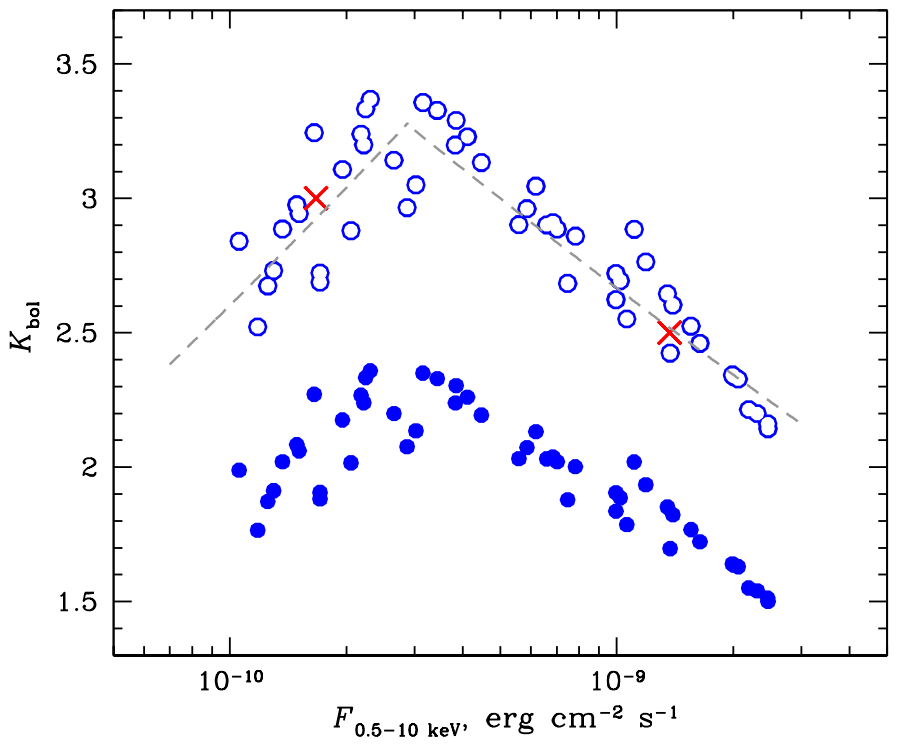

Fig. 1. Bolometric correction factor as a function of the source intensity (open circles). Filled circles show the dependence of the ratio of the total flux in $0.5-10 \mathrm{keV}$ plus $15-50 \mathrm{keV}$ to the $0.5-10 \mathrm{keV}$ flux as a tracer of the bolometric correction factor $\left(K_{\mathrm{bol}}\right)$. Red crosses show the $K_{\text {bol }}$ value and the source flux (in $0.5-10 \mathrm{keV}$ range) from the broadband spectra collected with the $N U S T A R$ observatory.

observation) were produced using online tools provided by the UK Swift Science Data Centre (Evans et al. 2009) ${ }^{1}$.

The spectra were grouped to have at least one count per bin and fitted using a simple power law model modified with the photoelectric absorption (PHABS $\times$ POWERLAW model in the XSPEC package) and Cash statistic (Cash 1979). To avoid any problems caused by the calibration uncertainties at low energies $^{2}$, we restricted the spectral analysis to the $0.7-10 \mathrm{keV}$ and 0.5-10 keV energy bands for the data in WT and PC modes, respectively.

We found that at all luminosities the spectra of SMCX-3 can be well described with a simple absorbed power-law model with the photon index being in the range of $0.5-1.1$. The spectral analysis did not reveal any significant absorption in excess of the Galactic hydrogen column density measured in this direction $N_{\mathrm{H}}=0.066 \times 10^{22} \mathrm{~cm}^{-2}$ (Dickey \& Lockman 1990). To make the spectral approximation more robust we fixed the $N_{\mathrm{H}}$ value at this value. Weng et al. (2017) claimed an appearance of the blackbody component at very soft energies which were ignored in our analysis.

\subsection{NUSTAR data}

The NUSTAR observatory consists of two co-aligned identical $\mathrm{X}$-ray telescope systems (FPMA and FPMB) operating in a wide energy range from 3 to $79 \mathrm{keV}$ (Harrison et al. 2013). Thanks to the unique multilayered mirrors, NuSTAR has an unprecedented sensitivity in hard X-rays $(>10 \mathrm{keV})$ and is ideally suited for the broadband spectroscopy of different objects, including X-ray pulsars, and searching for the CSRFs in their spectra.

SMC X-3 has been observed with NUSTAR twice during the current 2016-2017 outburst (ObsIDs 90201035002 and 90201041002) with the aim of measuring its hard X-ray spectrum. Preliminary results of the analysis of the first observation

\footnotetext{
http://www.swift.ac.uk/user_objects/

http://wWw.swift.ac.uk/analysis/xrt/digest_cal.php
} 
with the bolometric luminosity $\sim 10.2 \times 10^{38} \mathrm{erg} \mathrm{s}^{-1}$ were discussed above (Pottschmidt et al. 2016). The second observation was performed, at our request, three months later on MJD 57704.8 when the source luminosity was $\sim 1.9 \times 10^{38} \mathrm{erg} \mathrm{s}^{-1}$. This is about an order of magnitude lower than first observation.

The raw data obtained during both observations were processed to produce cleaned event files for the FPMA and FPMB modules using the standard NUSTAR Data Analysis Software (NUSTARDAS) v1.7.1 provided under HEASOFT V6.20 with the CALDB version 20170222. Using the NUPRODUCTS routine, we extracted the source spectra from the circlar region with radius of $120^{\prime \prime}$. The background spectrum was extracted in the region of the same radius located $5^{\prime}$ from the source position.

\subsection{Bolometric correction}

For any meaningful discussion of the observed source properties, a bolometric correction has to be estimated for the observed flux in soft energy band. To do that we used two available NUSTAR observations as reference points and the dependence of the ratio of the total source flux in the $0.5-10 \mathrm{keV}$ plus $15-$ $50 \mathrm{keV}$ energy bands to the source flux in the $0.5-10 \mathrm{keV}$ energy band. The flux in the $15-50 \mathrm{keV}$ band has been estimated using Swift/BAT transient monitor ${ }^{3}$ light curve of the source. The ratio $\left(F_{0.5-10 \mathrm{keV}}+F_{15-50 \mathrm{keV}}\right) / F_{0.5-10 \mathrm{keV}}$ as a function of $F_{0.5-10 \mathrm{keV}}$ is shown in Fig. 1. To convert this ratio to the bolometric correction factor $K_{\text {bol }}$ we rescaled it to match the values calculated from the spectral parameters obtained from two NUSTAR observations. The result of this adjustment was fitted with a broken linear model (shown in the same figure). We estimate that residual systematic uncertainty in the correction factor does not exceed $5 \%$. Due to the unknown shape of the source broadband spectrum at low fluxes we fixed the bolometric correction factor value at two for all observations with $F_{0.5-10 \mathrm{keV}}<4 \times 10^{-11} \mathrm{erg} \mathrm{s}^{-1} \mathrm{~cm}^{-2}$. In the following analysis we apply this correction to all observational data and refer to the bolometrically corrected fluxes and luminosities, unless stated otherwise.

\section{Results}

The light curve of SMC X-3 observed with the Swift/XRT telescope is shown in the upper panel of Fig. 2 with black points. The source flux was estimated using the bolometric and absorption corrections described above and assuming a distance to the source of $62 \mathrm{kpc}$ (Haschke et al. 2012). The observed light curve looks rather complicated. One can see from Fig. 2 a clear transition to a faster luminosity decay after MJD $\sim 57710$. This transition can be understood in terms of the thermal-viscous instability model as a moment when temperature at the outer radius of the accretion disk reached the critical temperature of $\sim 6500 \mathrm{~K}$ causing the decrease of the local viscosity and corresponding a decline of the mass accretion rate onto the compact object (see, e.g., Lasota 2001). This results in the fast decay of the luminosity observed, particularly, from XRPs in the very end of their outbursts (see recent works by Tsygankov et al. 2016a; Lutovinov et al. 2017). As can be seen from Fig. 2 this decay is not smooth and was interrupted with temporal re-brightenings a few times on MJD 57 730, MJD 57770 and MJD 57810 , near the corresponding periastron passages.

\footnotetext{
http://swift.gsfc.nasa.gov/results/transients/
}

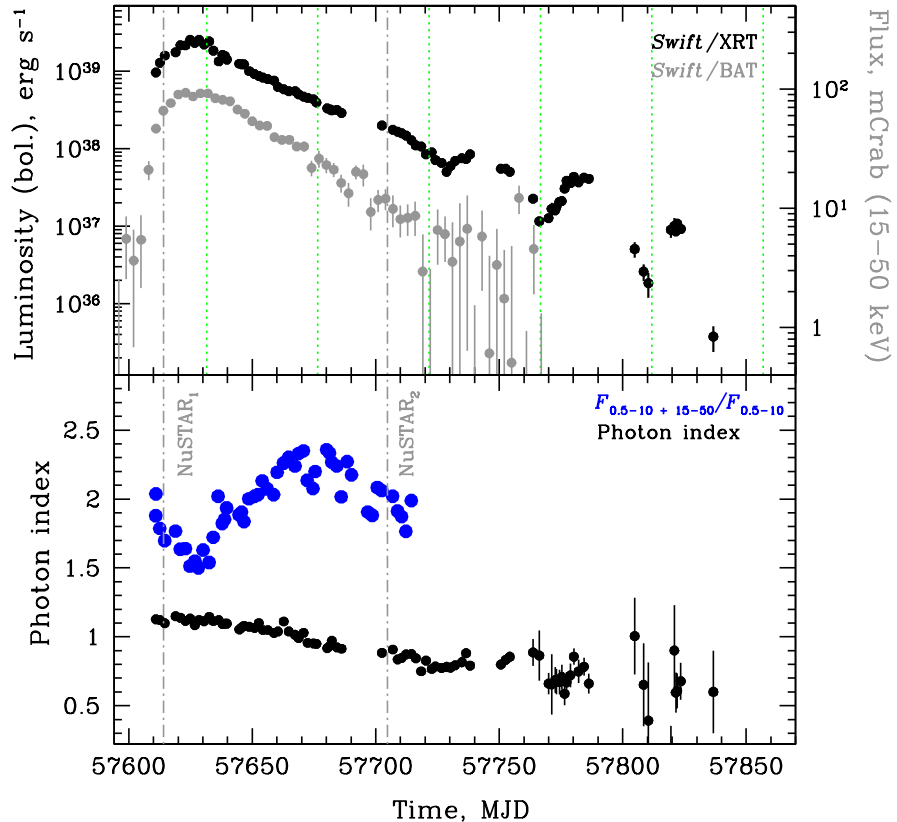

Fig. 2. Upper panel: bolometric light curve of SMC X-3 obtained with the Swift/XRT telescope (black points). Luminosity is calculated from the unabsorbed flux under assumption of the distance to the source $d=62 \mathrm{kpc}$ and bolometric correction factors from Fig. 1. Gray dots represent flux in the $15-50 \mathrm{keV}$ band from the $S$ wift/BAT monitor (in the units of mCrab, right axis). Green vertical dotted lines correspond to the times of the periastron passages. Bottom panel: evolution of the photon index and ratio of fluxes $\left(F_{0.5-10 \mathrm{keV}}+F_{15-50 \mathrm{keV}}\right) / F_{0.5-10 \mathrm{keV}}$ over the outburst are shown with black and blue points, respectively. Vertical dash-dotted lines correspond to the times of the NuSTAR observations.

\subsection{Orbital parameters}

To determine the pulse frequency of the source we used the XRT window-timing mode event data which has sufficient counting statistics and time resolution. After applying the standard filtering criteria, we selected events with energies $0.3-10 \mathrm{keV}$ from the source-centered circle with a radius of 25 pixels and applied barycentric correction to the photon arrival times. To determine the spin frequency of the source for each observation we performed a search for significant peaks around the source pulse period using the H-test (de Jager et al. 1989). To estimate the uncertainty of the obtained value we used the same approach as Markwardt et al. (2002), that is, we assumed that one sigma uncertainty for the most significant frequency peak in periodogram corresponds to the drop $\Delta Z^{2}=1$ with respect to the peak value. The results are presented in Fig. 3 and are consistent with values reported by Fermi/GBM pulsar project ${ }^{4}$ (Townsend et al. 2017; Weng et al. 2017).

We note that modulation of the pulse frequency associated with the orbital motion is apparent and has to be taken into the account when determining the intrinsic spin frequency of the NS. The parameters of the binary orbit were estimated for the current 2016-2017 outburst by Townsend et al. (2017) and Weng et al. (2017). The discrepancies, particularly in the obtained orbital period value, likely arise due to the difficulties in modeling of complex intrinsic spin evolution of the source.

To obtain an improved orbital solution we followed an approach similar to that of Townsend et al. (2017), that is, we estimated the intrinsic spin frequency of the pulsar based on the

\footnotetext{
4 https://gammaray.nsstc.nasa.gov/gbm/science/pulsars/ lightcurves/smcx3.html
} 
Table 1. Best-fit orbital parameters of SMC X-3.

\begin{tabular}{cc}
\hline \hline Parameter & Value \\
\hline Orbital period $(\mathrm{d})$ & $45.07(5)$ \\
$a_{x} \sin i$ (light seconds) & $189(1)$ \\
$e$ & $0.231(6)$ \\
$\omega$ (degrees) & $206(2)$ \\
$T_{\omega}$ (MJD) & $57631.5(2)$ \\
$\chi^{2}$ d.o.f. & $117.2 / 91$ \\
\hline
\end{tabular}

Notes. Parameters are based on the full sample of the Fermi/GBM and Swift/XRT data. Uncertainties are quoted at $1 \sigma$ confidence level including the model systematics. The fit statistic for the same parameters without inclusion of the model systematics is $\chi^{2}=3992$ for 91 d.o.f.

expected accretion-induced spin-up. We note that angular momentum transferred to the NS by the accretion disk is proportional to the accretion rate and thus is expected to dominate the spin evolution of the NS at high luminosities. The details of interaction of the disk with the magnetosphere are thus not very important and it is sufficient to consider the accelerating torque only (which, however, still depends on the magnetosphere size) with the spin-up rate defined as (Lipunov 1982)

$\dot{v}=\dot{M} \sqrt{G M R_{\mathrm{d}}} / 2 \pi I$,

where the inner disk radius $R_{\mathrm{d}}=k R_{\mathrm{A}}$ is assumed to constitute some fraction of the Alfvénic radius, and $I$ is NS moment of inertia. We note that this is essentially the same model as that used by Townsend et al. (2017). The main difference is that we use the bolometric light curve rather than flux in the soft band to estimate the accretion rate $\dot{M}=R_{\mathrm{NS}} L_{\mathrm{X}} / G M$. Another difference is that we also included the spin frequency measurements reported by Fermi/GBM into the fit to improve statistics. Finally, Townsend et al. (2017) do not account for the model systematics associated with the fact that uncertainties in the observed accretion rate inevitably translate to a systematic uncertainty in the predicted frequency when integrating over the outburst. On the other hand, this uncertainty can be easily estimated directly from the dispersion of model predictions for a set of light curves simulated based on the observed fluxes and uncertainties (once initial estimate for $R_{\mathrm{d}}$ is obtained). We estimated the model systematics to increase from zero at the beginning of the outburst to $\sim 2 \times 10^{-6} \mathrm{~Hz}$ at the end of the outburst, and added it in quadrature to the statistical uncertainties to obtain the final fit and estimate the uncertainties for the orbital parameters presented in Table 1. We note that using the bolometric light curve allows us to obtain a much better fit than that reported by Townsend et al. (2017) even without accounting for model systematics.

\subsection{Pulse profile and pulsed fraction}

Excellent time and energy resolutions of the NUSTAR observatory allowed us to investigate temporal properties of SMC X-3 at energies above $20 \mathrm{keV}$ in details for the first time. Moreover, two observations performed on the rising and declining parts of the outburst gave us a possibility to trace evolution of these properties at different mass accretion rates.

The source pulse profiles in six energy bands from 3 to $79 \mathrm{keV}$ are shown in Fig. 4 for two luminosities: $L_{\mathrm{bol}}=10.2 \times$ $10^{38} \mathrm{erg} \mathrm{s}^{-1}$ (top panel; ObsID 90201035002) and $L_{\text {bol }}=1.9 \times$ $10^{38} \mathrm{erg} \mathrm{s}^{-1}$ (bottom panel; ObsID 90201041002). To determine the pulse period and to produce the pulse profile we used the combined light curves from both modules in order to obtain the

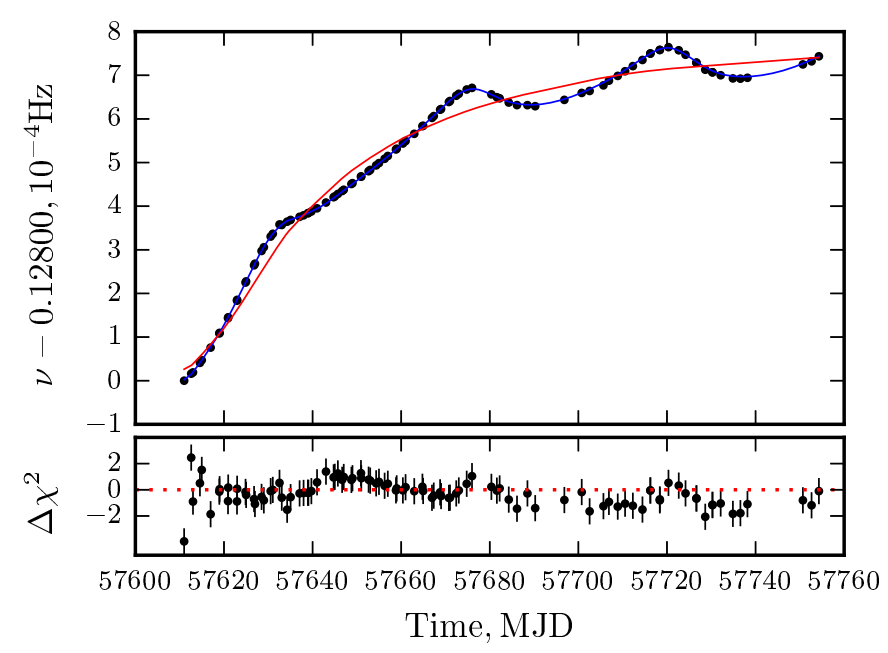

Fig. 3. Spin evolution of SMCX-3 as observed by Fermi/GBM and Swift/BAT (black points), and the best-fit models for intrinsic (red) and observed (blue) pulse frequencies. Residuals for the best-fit model are also shown in the bottom panel.

better statistics (see Krivonos et al. 2015, for the description of the procedure). To produce the pulse profile in some energy range the corresponding light curve was folded with the corrected for the orbital motion spin periods $P_{\mathrm{s}}=7.80954(5) \mathrm{s}$ and $P_{\mathrm{s}}=7.77084(7) \mathrm{s}$ for first and second observations, respectively.

In both luminosity states the pulse profile exhibits a simple double-peak shape with peaks separated by a half phase. The main difference is that the intensity of both peaks is approximately the same in the high state, whereas in the observation with the lower luminosity the intensity of the first (main) peak is significantly higher. The energy dependence is very weak and noticeable mainly in the second observation. Namely, the main peak (at phase $\sim 0.25$ ) has a double-peak substructure at low energies, gradually changing to a single-peak with the energy increase. At the same time, the second peak (at phase $\sim 0.75$ ) is disappearing at higher energies.

The pulsed fraction calculated using the standard definition $^{5}$ as a function of the energy is presented in Fig. 5. For both observations the pulsed fraction is increasing toward higher energies, that is typical for the majority of X-ray pulsars (Lutovinov \& Tsygankov 2009). We note that during the observation with the higher luminosity the pulsed fraction was significantly higher throughout the energy range.

As can be seen from Figs. 4 and 5 the pulsed fraction is around $100 \%$ at hard X-rays during the first observation. This is a very important fact since it rules out the presence of a strong geometrical beaming of the emission by the accretion disk, often used to artificially decrease the mass accretion rate onto the NS in pulsing ULXs (see e.g., King et al. 2017). Another characteristic of the pulsed fraction dependence on the energy is its smoothness. It is consistent with the absence of any strong features (e.g., CSRF) in the source energy spectrum (see Sect. 3.3). Indeed, the non-monotonic dependence of the pulsed fraction on energy around the cyclotron line was shown to be typical for a few X-ray pulsars (Tsygankov et al. 2006; Lutovinov \& Tsygankov 2009; Ferrigno et al. 2009).

\footnotetext{
$5 \mathrm{PF}=\left(F_{\max }-F_{\min }\right) /\left(F_{\max }+F_{\min }\right)$, where $F_{\max }$ and $F_{\min }$ are maximum
} and minimum fluxes in the pulse profile, respectively. 


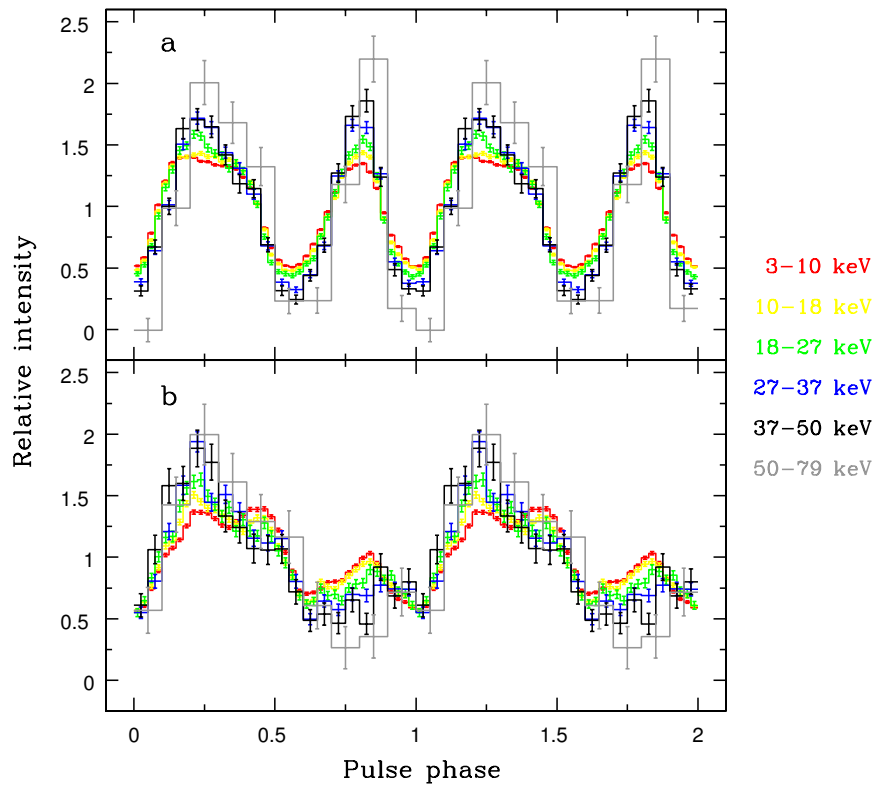

Fig. 4. Dependence of the pulse profile of SMCX-3 on energy in two NUSTAR observations. Different panels correspond to different X-ray luminosities: a) $L_{\mathrm{bol}}=10.2 \times 10^{38} \mathrm{erg} \mathrm{s}^{-1}$ (ObsID 90201035002), b) $L_{\text {bol }}=1.9 \times 10^{38} \mathrm{erg} \mathrm{s}^{-1}$ (ObsID 90201041002). Different energy bands are shown with different colors (shown on the right). The profiles are normalized by the mean flux in each energy band and plotted twice for clarity.

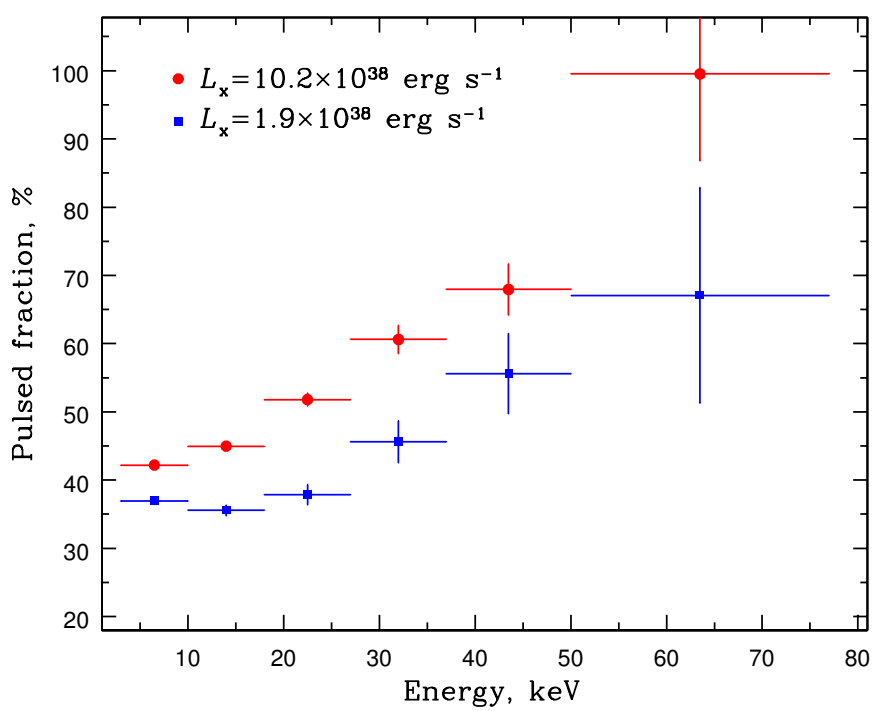

Fig. 5. Dependence of the pulsed fraction on energy in two NuSTAR observations with different X-ray luminosities: $L_{\mathrm{bol}}=10.2 \times 10^{38} \mathrm{erg} \mathrm{s}^{-1}$ (red circles; ObsID 90201035002) and $L_{\mathrm{bol}}=1.9 \times 10^{38} \mathrm{erg} \mathrm{s}^{-1}$ (blue squares; ObsID 90201041002).

\subsection{Spectroscopy}

To supplement the NUSTAR data in the soft energy band and to determine better the broadband spectrum of SMC X-3 we used the nearest in time Swift/XRT observations (ObsIDs 00034673002 and 00034673045 , respectively). The broadband spectrum in the high luminosity state was described with the same model as used by Pottschmidt et al. (2016) with following best-fit parameters values: the photon index $\Gamma=0.89 \pm 0.01$, the folding energy $E_{\text {fold }}=14.88 \pm 0.14 \mathrm{keV}$, the blackbody temperature $k T=0.89 \pm 0.05 \mathrm{keV}$, the iron line energy $6.38 \pm 0.03 \mathrm{keV}$

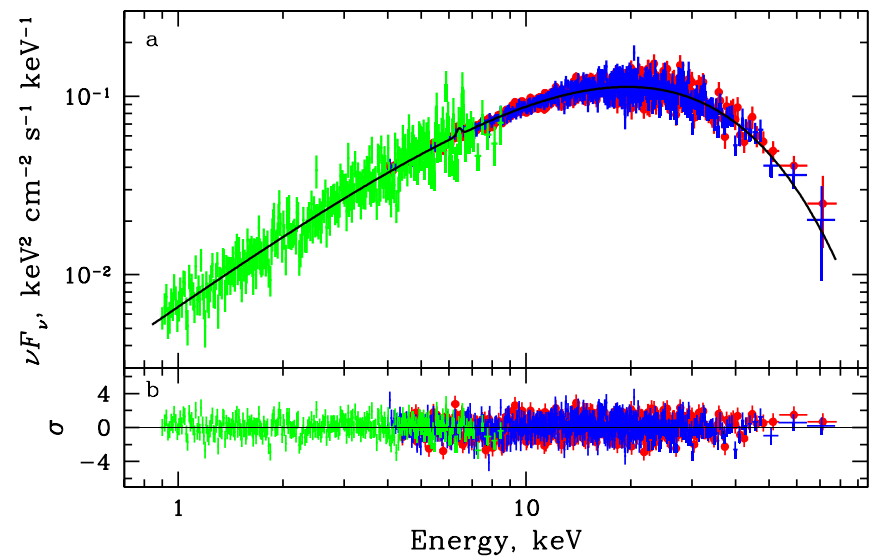

Fig. 6. Upper panel: broadband spectrum of SMCX-3 measured with Swift/XRT telescope (green points; ObsID 00034673045) and two NUSTAR modules FPMA and FPMB (red and blue points, respectively; ObsID 90201041002). The black solid line represents the best fit model consisting of exponentially cutoff power-law with addition of the fluorescent iron line. Lower panel: corresponding residuals to the best-fit model.

and width $0.37 \pm 0.05 \mathrm{keV}$ (the equivalent width of $69 \pm 5 \mathrm{eV}$ ). We note that the continuum parameters are somewhat different from those reported by Pottschmidt et al. (2016) due to the inclusion of the XRT data in our analysis. The spectrum in the low luminosity state can be well approximated by the exponentially cutoff power-law with photon index of $\Gamma=0.62 \pm 0.01$ and folding energy of $E_{\text {fold }}=14.16 \pm 0.17 \mathrm{keV}$. A fluorescent iron line at the energy of $6.40 \pm 0.13 \mathrm{keV}$ with the equivalent width of $18 \pm 8 \mathrm{eV}$ is also tentatively registered in the spectrum. Because the statistic was not good enough to determine the line width directly, it was fixed at the value 0.37 , determined from the first observations, that roughly corresponded to the NUSTAR energy resolution (Harrison et al. 2013). No thermal component is required for the second observation. The corresponding broadband spectrum of the source is presented in Fig. 6. To take into account the uncertainty in the instrument calibrations as well as the lack of strict simultaneity of observations by NUSTAR and Swift, cross-calibration constants between them were included in the spectral modeling.

No other obvious spectral features (primarily, the CSRF) were found in both NUSTAR spectra of SMCX-3. To quantify this conclusion we used an approach initially proposed by Tsygankov \& Lutovinov (2005) and recently improved by Shtykovsky et al. (2017). The spectral models were modified through addition of the GABS component from the XSPEC package. The CSRF energy $E_{\text {cyc }}$ was varied within the 6-62 keV energy range with the step of $3 \mathrm{keV}$. A corresponding line width was varied within the $4-8 \mathrm{keV}$ range with the step of $2 \mathrm{keV}$. Each combination of line's position and width were fixed and the resulting model was used to approximate the source spectrum. As a result, none of these combinations resulted in a significant improvement of the fit and only the upper limits for the optical depth of $\sim 0.25$ and $\sim 0.19(3 \sigma)$ can be obtained for the first and second NUSTAR observations, respectively.

Finally, we also performed a pulse phase-resolved spectroscopy of the emission of SMC X-3 using the NuSTAR data. It was found that the source spectrum at different phases is well described by the same models as were used for the average spectra. An example of the source's spectral parameters variations over the pulse is shown in Fig. 7 for the high luminosity state. It is clearly seen that all continuum parameters are slightly varying 


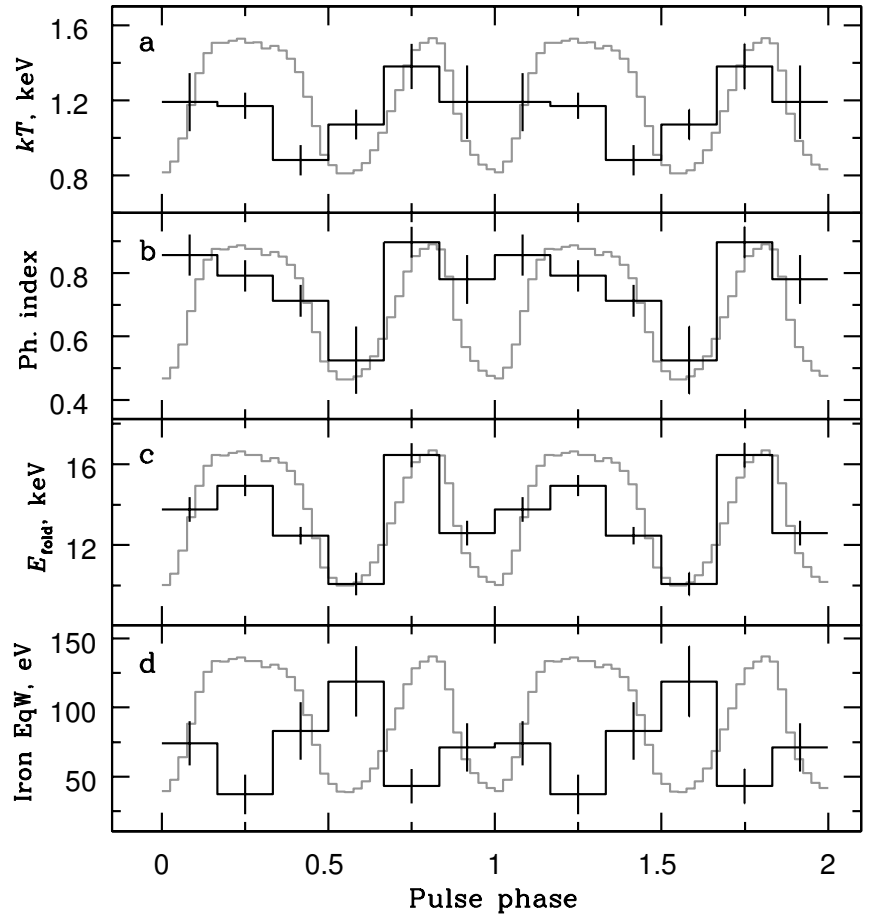

Fig. 7. Variations of spectral parameters of SMCX-3 over the pulse phase for the high luminosity state (NuSTAR observation ID 90201035002). The black histogram in the panels represents: $a$ ) blackbody temperature, $b$ ) photon index, $c$ ) folding energy and $d$ ) equivalent width of the iron emission line. The gray line in each panel shows the pulse profile in a wide energy range.

with the pulse phase in the ranges of $\Gamma \sim 0.5-0.9, E_{\text {fold }} \sim 11-$ $16 \mathrm{keV}, k T \sim 0.9-1.3 \mathrm{keV}$. It is interesting to note that the equivalent width of the iron emission line is also variable with the phase, demonstrating a possible anti-correlation with the pulse intensity. Such a behavior has already been reported for several other sources (see, e.g. Tsygankov \& Lutovinov 2009; Shtykovsky et al. 2017) and can be used for the tomography of the matter distribution in the vicinity of the NS. For the low luminosity state the behavior of parameters is quite similar: the photon index and folding energy vary with the pulse phase in the ranges of $\Gamma \sim 0.5-0.7$ and $E_{\text {fold }} \sim 11-17 \mathrm{keV}$, respectively. The emission iron line is significantly detected only in one of the phase bins. Again, no indications for the CSRF were found in these spectra.

\section{Discussion}

\subsection{Critical and maximal luminosities}

In the case of very strong magnetic field Compton scattering cross section is strongly reduced (see, e.g., Mushtukov et al. 2016). This reduces an impact of the radiative pressure and was shown to be required to allow the accreting X-ray pulsar to exhibit super-Eddington luminosities during giant outbursts similar to one observed from SMC X-3. Specifically, this is possible due to the rise of the accretion column above the NS surface (Basko \& Sunyaev 1976; Mushtukov et al. 2015b). Critical luminosity $L^{*}$ dividing two regimes of accretion is a function of the magnetic field strength in the vicinity of the NS surface. Therefore, observational constraint of $L^{*}$ can be used to obtain independent estimates of the magnetic field. It is worth noting here that observational evidence of a transition through the critical luminosity was recently found in the classical XRP V 0332+53 (Doroshenko et al. 2017).

It is interesting that SMCX-3 exhibits a substantial change of its pulse profile between MJD $\sim 57680$ and MJD $\sim 57700$ when its main peak shifted by $\sim \pi / 2$ (see Fig. 4 in Weng et al. 2017). We argue that this change happend when the bolometric luminosity of the source was $\sim(2-3) \times 10^{38} \mathrm{erg} \mathrm{s}^{-1}$ is caused by the disappearance of the accretion column and corresponding modification of the intrinsic X-ray beaming from the pulsar (Gnedin \& Sunyaev 1973). Interpretation of this luminosity as a critical one results in the estimates of the magnetic field of $B \simeq(2-3) \times 10^{13} \mathrm{G}$ (Mushtukov et al. 2015b).

Maximal accretion luminosity of the NS depends on the magnetic field strength as well (Mushtukov et al. 2015a). The bolometric peak luminosity of SMCX-3 achieved during the 2016-2017 outburst is $\sim 2.5 \times 10^{39} \mathrm{erg} \mathrm{s}^{-1}$ exceeding the Eddington limit by an order of magnitude. According to the accretion column theory by Mushtukov et al. (2015a) so high luminosity is impossible if the NS magnetic field is less than $\sim 2 \times 10^{13} \mathrm{G}$. This value agrees well with the one derived above from the critical luminosity. Both methods refer to the magnetic field strength in the region of the main energy release - in the immediate vicinity of the NS surface.

\subsection{Accretion torque}

Evolution of the intrinsic spin frequency of accreting pulsars is driven by angular momentum transfer from the accretion disk, and possibly by braking mechanisms associated with interaction of the magnetosphere with the disk (Ghosh \& Lamb 1979; Wang 1987; Lovelace et al. 1995; Parfrey et al. 2016). In the case of SMC X-3, however, the accelerating torque is expected to dominate, and indeed, as already mentioned in Sect. 3.1, no evidence for braking has been observed. The observed spin evolution is thus fully consistent with pure spin-up by the disk which is trivial to calculate and allows estimation of the magnetic field. As follows from Eq. (1), the magnetosphere size or, equivalently, magnetic field strength of the NS is one of the model parameters that can be obtained during the fit of the orbital parameters if physical parameters of the NS and relation of the inner disk and Alfvénic radii are known or assumed. For a standard NS with mass and radius of $1.4 M_{\odot}$ and $10 \mathrm{~km}$ respectively, and coupling factor $k=0.5$ the field strength is well constrained at $B=2.55(1) \times 10^{12} \mathrm{G}$. This is consistent with the value reported by Klus et al. (2014) based on the spin evolution between outbursts, and an order of magnitude lower than that estimated above based on the observed critical and maximal luminosities.

\subsection{Propeller effect}

The distinctive property of accretion onto the highly magnetized NS is a strong centrifugal barrier produced by the rotating magnetosphere. This barrier does not allow the accreting matter to penetrate into the magnetosphere if the velocity of the field lines is higher than corresponding local Keplerian velocity, that is known as propeller effect (Illarionov \& Sunyaev 1975; Stella et al. 1986). In other words, the accretion is only possible if the magnetospheric radius $R_{\mathrm{m}}$ does not exceed the co-rotation radius $R_{\mathrm{c}}$.

Because the magnetospheric radius depends on the mass accretion rate, we can link the transition luminosity $L_{\text {lim }}$ with the spin period of the NS and its magnetic field strength. The corresponding equation can be derived by the equating 


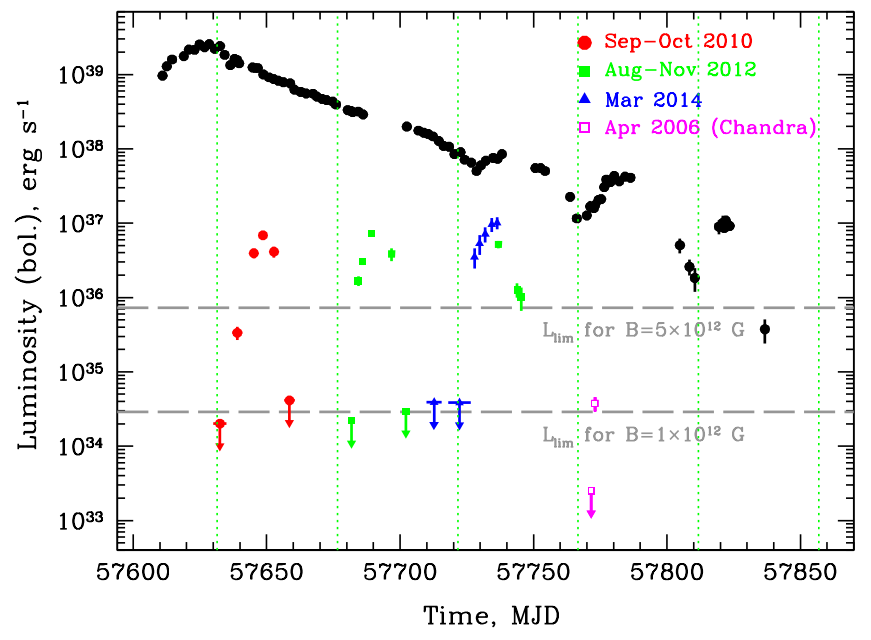

Fig. 8. Bolometric light curve of SMCX-3 as seen by the Swift/XRT telescope obtained during the giant outburst in 2016-2017, type I outbursts in September-October 2010, August-November 2012 and March 2014 are shown with black circles, red circles, green squares and blue triangles, respectively. The Chandra measurement from April 2006 is represented by a magenta square. The $3 \sigma$ upper limits are shown with arrows of the corresponding color. The historical data were shifted by an integer number of the orbital periods obtained in the current work (see Table 1) for illustrative purpose. The luminosity is calculated from the unabsorbed flux under assumption of the distance to the source $d=62 \mathrm{kpc}$ and bolometric correction factors from Fig. 1. The horizontal dashed lines show the upper and lower limits for the threshold luminosity for the propeller regime onset (see the text). Green vertical dotted lines correspond to the times of the periastron passages.

the magnetospheric radius to the co-rotation radius (e.g., Campana et al. 2002):

$L_{\mathrm{lim}}(R) \simeq \frac{G M \dot{M}_{\mathrm{lim}}}{R} \simeq 4 \times 10^{37} k^{7 / 2} B_{12}^{2} P^{-7 / 3} M_{1.4}^{-2 / 3} R_{6}^{5} \mathrm{erg} \mathrm{s}^{-1}$,

where $P$ is the NS spin period in seconds, $B_{12}$ is the strength of the dipole component of the magnetic field in units of $10^{12} \mathrm{G}$, $M_{1.4}$ and $R_{6}$ are the NS mass and radius in units of $1.4 M_{\odot}$ and $10^{6} \mathrm{~cm}$, respectively. A factor $k$ relates the magnetospheric radius to the classical Alfvén radius in the case of disk accretion and is usually taken $k=0.5$ (Ghosh \& Lamb 1978).

On 2017 February 1, SMC X-3 was still very bright to expect the transition to the propeller regime for the NS with standard magnetic field. Therefore, we investigated the archival Swift/XRT and Chandra data. The archival Swift observations were analyzed as described above. The source spectra available as part of the Chandra Source Catalog ${ }^{6}$ were fit using the XSPEC and the same model as for the Swift/XRT spectra. The resulting light curves based on all available data as well as $3 \sigma$ upper limits are shown in Fig. 8.

From Fig. 8 one can see that SMCX-3 was significantly detected at low luminosities during several type I outbursts. The faintest state of the source with bolometric luminosity of $\sim 3.5 \times 10^{34} \mathrm{erg} \mathrm{s}^{-1}$ was observed by the Chandra observatory on 2006, April 26. Just one day before that, the source was not detected with a $3 \sigma$ upper limit of $\sim 2.5 \times 10^{33} \mathrm{erg} \mathrm{s}^{-1}$. The lowest significant luminosity of SMC X-3 can serve as an estimate for the threshold of the propeller regime onset $L_{\mathrm{lim}} \sim 3 \times 10^{34} \mathrm{erg} \mathrm{s}^{-1}$. On the other hand, based only on the Swift/XRT data the limiting luminosity can be as high as $L_{\mathrm{lim}} \sim 7 \times 10^{35} \mathrm{erg} \mathrm{s}^{-1}$.

\footnotetext{
6 http://cxc.harvard. edu/csc/; Evans et al. (2010).
}

Therefore, we use here a very conservative range of luminosities from $\sim 3 \times 10^{34} \mathrm{erg} \mathrm{s}^{-1}$ to $\sim 7 \times 10^{35} \mathrm{erg} \mathrm{s}^{-1}$ to estimate the dipole component of the magnetic field (shown in Fig. 8). Substituting the measured spin period of SMC X-3 $(P=7.81 \mathrm{~s})$ and the range of limiting propeller luminosity $L_{\text {lim }}$ to Eq. (2) and assuming the standard mass and radius of the NS we can estimate the dipole component of the NS magnetic field as $B \sim(1-5) \times 10^{12} \mathrm{G}$.

Interestingly, both methods (accretion torque and the propeller effect) measuring the dipole component of the magnetic field agree well with each other and give significantly lower strengths than the methods sensitive to the magnetic field in the vicinity of the NS (critical and maximal luminosities). This fact suggests the strongly non-dipole configuration of the NS magnetic field with multipoles by an order of magnitude stronger than dipole component within the emission region. It is worth mentioning that absence of the CSRF is consistent with the above picture. Another possibility for resolving this discrepancy is the significant deviation of the parameter $k$ from the standard value of 0.5 adopted in our study. Specifically, the estimate of the dipole component of the magnetic field $B_{\text {dipole }}$ will coincide with multipole component $B_{\text {multipole }}$ for $k \approx 0.15$.

However, this assumption seems to be inconsistent with the results obtained previously for other sources. Indeed, to date the propeller effect was observed in SAX J1808.4-3658 (Campana et al. 2008), GRO J1744-28 (Cui 1997), 4U 0115+63 (Tsygankov et al. 2016a), V 0332+63 (Tsygankov et al. 2016a), SMCX-2 (Lutovinov et al. 2017), and the accreting magnetar M82 X-2 (Tsygankov et al. 2016b). All these sources are collected in Fig. 9 where the combination of the propeller limiting luminosity and corresponding pulse period, $L_{\lim } P^{7 / 3}$, are compared with magnetic field strength measured independently (mainly based on the observed CSRF energy, see Tsygankov et al. 2016a, and references therein). We note that for all six sources the limiting luminosity seems to agree with the theoretical dependence given by Eq. (2) for $k=0.5$ shown with dashed line. Discrepancy between two field components was also pointed out in two pulsating ULXs in NGC 5907 (Israel et al. 2017a) and NGC 7793 P13 (Israel et al. 2017b). Interestingly, in both sources multipole components are estimated to be an order of magnitude stronger in comparison to the dipole component (see green circles in Fig. 9), similarly to the case of SMC X-3.

\section{Conclusion}

In this paper we report the estimate of the magnetic field strength in a bright X-ray pulsar SMC X-3 which can be considered the closest ULX-pulsar. The source exhibited a giant outburst in July 2016-March 2017 with the peak bolometric luminosity of $\sim 2.5 \times 10^{39} \mathrm{erg} \mathrm{s}^{-1}$. The entire outburst had been monitored with the Swift/XRT and Fermi/GBM telescopes, as well as with the NUSTAR observatory. The collected data allowed us to estimate the magnetic field strength of the NS in SMC X-3 using several independent methods based on the spectral and timing properties of X-ray emission from the system.

The dipole component of the magnetic field was determined using the accretion torque models and observation of the transition to the propeller regime at the limiting luminosity in the range of $\sim(0.3-7) \times 10^{35} \mathrm{erg} \mathrm{s}^{-1}$ both resulting in a relatively weak strength of about $(1-5) \times 10^{12} \mathrm{G}$. On the other hand, there is evidence for a much stronger field strength in the immediate vicinity of the NS surface. In particular the transition from superto sub-critical accretion regime associated with cease of an accretion column and very high peak luminosity favor the magnetic 


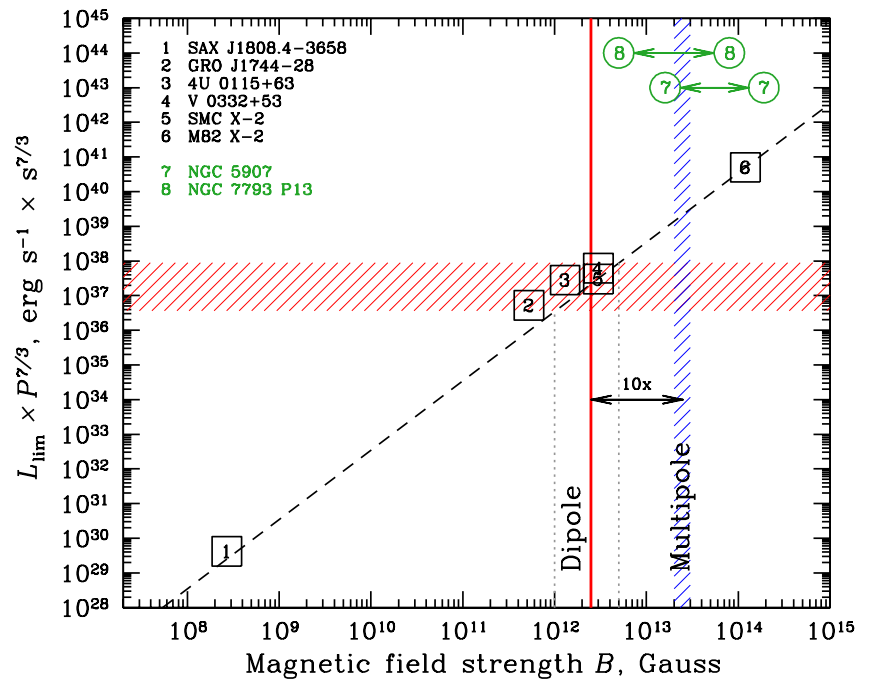

Fig. 9. Correlation between a combination of the propeller limiting luminosity and the pulsar spin period, $L_{\mathrm{lim}} P^{7 / 3}$, and independently determined magnetic field strength $B$ for six pulsating sources (black squares; adopted from Tsygankov et al. 2016a). The dashed line represents the theoretical dependence given by Eq. (2) for $k=0.5$. Estimate of the limiting luminosity and corresponding dipole field strength for SMC X-3 are shown with horizontal shaded region and dotted vertical lines, respectively. The solid red vertical line indicates the dipole field estimate from the observed spin evolution of the source. Constraints on the field strength in vicinity of the NS based on the observed critical and maximal luminosities are shown with vertical shaded region. We note an order of magnitude discrepancy between the field estimates at the magnetosphere and in vicinity of the NS. Green circles show similar discrepancy between two field components in the pulsating ULXs in NGC 5907 and NGC 7793 P13.

field of $\sim(2-3) \times 10^{13} \mathrm{G}$. The absence of the CSRF in the broadband X-ray spectrum of the source obtained with NUSTAR is also consistent with this estimate.

This discrepancy makes SMC X-3 a good candidate to be a NS with strong multipole configuration of the magnetic field, and an intermediate source between classical X-ray pulsars and accreting magnetars which may constitute an appreciable fraction of ULX population. Alternatively, this discrepancy can be resolved if one assumes that the accretion disk in SMCX-3 pushes much deeper into magnetosphere than normally expected. However, the reason for such behavior would be unclear as it was not observed in other objects with wide range of luminosities for which similar analysis was conducted.

On the other hand, existence of higher field multipoles has also been claimed for another pulsating ULXs (Ekşi et al. 2015; Israel et al. 2017a,b). One can thus speculate that a complex structure of the NS magnetic field may thus be a common feature for this class of objects.

Acknowledgements. This work was supported by the Russian Science Foundation grant 14-12-01287 (S.S.T., A.A.L., A.A.M.), the Foundations' Professor Pool, the Finnish Cultural Foundation and the Academy of Finland grant 268740 (J.P.). We also acknowledge the support from the COST Action MP1304.

\section{References}

Basko, M. M., \& Sunyaev, R. A. 1976, MNRAS, 175, 395 Burrows, D. N., Hill, J. E., Nousek, J. A., et al. 2005, Space Sci. Rev., 120, 165
Campana, S., Stella, L., Israel, G. L., et al. 2002, ApJ, 580, 389 Campana, S., Stella, L., \& Kennea, J. A. 2008, ApJ, 684, L99 Cash, W. 1979, ApJ, 228, 939

Clark, G., Doxsey, R., Li, F., Jernigan, J. G., \& van Paradijs, J. 1978, ApJ, 221, L37

Corbet, R. H. D., Laycock, S., Coe, M. J., Marshall, F. E., \& Markwardt, C. B. 2004, in X-ray Timing 2003: Rossi and Beyond, eds. P. Kaaret, F. K. Lamb, \& J. H. Swank, AIP Conf. Ser., 714, 337

Cowley, A. P., \& Schmidtke, P. C. 2004, AJ, 128, 709

Crampton, D., Hutchings, J. B., \& Cowley, A. P. 1978, ApJ, 223, L79

Cui, W. 1997, ApJ, 482, L163

de Jager, O. C., Raubenheimer, B. C., \& Swanepoel, J. W. H. 1989, A\&A, 221, 180

Dickey, J. M., \& Lockman, F. J. 1990, ARA\&A, 28, 215

Doroshenko, V., Tsygankov, S. S., Mushtukov, A. A., et al. 2017, MNRAS, 466, 2143

Edge, W. R. T., Coe, M. J., Galache, J. L., et al. 2004, MNRAS, 353, 1286

Ekşi, K. Y., Andaç, İ. C., Çıkıntoğlu, S., et al. 2015, MNRAS, 448, L40

Evans, P. A., Beardmore, A. P., Page, K. L., et al. 2009, MNRAS, 397, 1177

Evans, I. N., Primini, F. A., Glotfelty, K. J., et al. 2010, ApJS, 189, 37

Ferrigno, C., Becker, P. A., Segreto, A., Mineo, T., \& Santangelo, A. 2009, A\&A, 498, 825

Gehrels, N., Chincarini, G., Giommi, P., et al. 2004, ApJ, 611, 1005

Ghosh, P., \& Lamb, F. K. 1978, ApJ, 223, L83

Ghosh, P., \& Lamb, F. K. 1979, ApJ, 234, 296

Gnedin, Y. N., \& Sunyaev, R. A. 1973, A\&A, 25, 233

Harrison, F. A., Craig, W. W., Christensen, F. E., et al. 2013, ApJ, 770, 103

Haschke, R., Grebel, E. K., \& Duffau, S. 2012, AJ, 144, 107

Illarionov, A. F., \& Sunyaev, R. A. 1975, A\&A, 39, 185

Israel, G. L., Belfiore, A., Stella, L., et al. 2017a, Science, 355, 817

Israel, G. L., Papitto, A., Esposito, P., et al. 2017b, MNRAS, 466, L48

Kennea, J. A., Coe, M. J., \& Evans, P. A. 2016a, ATel, 9370

Kennea, J. A., Coe, M. J., Evans, P. A., et al. 2016b, ATel, 9362

King, A., Lasota, J.-P., \& Kluźniak, W. 2017, MNRAS, 468, L59

Klus, H., Ho, W. C. G., Coe, M. J., Corbet, R. H. D., \& Townsend, L. J. 2014 MNRAS, 437, 3863

Krivonos, R. A., Tsygankov, S. S., Lutovinov, A. A., et al. 2015, ApJ, 809, 140

Lasota, J.-P. 2001, New A Rev., 45, 449

Lipunov, V. M. 1982, AZh, 59, 888

Lovelace, R. V. E., Romanova, M. M., \& Bisnovatyi-Kogan, G. S. 1995, MNRAS, 275, 244

Lutovinov, A. A., \& Tsygankov, S. S. 2009, Astron. Lett., 35, 433

Lutovinov, A. A., Tsygankov, S. S., Krivonos, R. A., Molkov, S. V., \& Poutanen, J. 2017, ApJ, 834, 209

Markwardt, C. B., Swank, J. H., Strohmayer, T. E., in 't Zand, J. J. M., \& Marshall, F. E. 2002, ApJ, 575, L21

Mushtukov, A. A., Suleimanov, V. F., Tsygankov, S. S., \& Poutanen, J. 2015a, MNRAS, 454, 2539

Mushtukov, A. A., Suleimanov, V. F., Tsygankov, S. S., \& Poutanen, J. 2015b, MNRAS, 447, 1847

Mushtukov, A. A., Nagirner, D. I., \& Poutanen, J. 2016, Phys. Rev. D, 93, 105003

Negoro, H., Nakajima, M., Kawai, N., et al. 2016, ATel, 9348

Parfrey, K., Spitkovsky, A., \& Beloborodov, A. M. 2016, ApJ, 822, 33

Pottschmidt, K., Ballhausen, R., Wilms, J., et al. 2016, ATel, 9404

Shtykovsky, A. E., Lutovinov, A. A., Arefiev, V. A., et al. 2017, Astron. Lett. 43, 175

Stella, L., White, N. E., \& Rosner, R. 1986, ApJ, 308, 669

Townsend, L. J., Kennea, J. A., Coe, M. J., et al. 2017, MNRAS, 471, 3878

Tsygankov, S. S., \& Lutovinov, A. A. 2005, Astron. Lett., 31, 88

Tsygankov, S., \& Lutovinov, A. 2009, in Proc. The Extreme Sky: Sampling the Universe above $10 \mathrm{keV}, 70$

Tsygankov, S. S., Lutovinov, A. A., Churazov, E. M., \& Sunyaev, R. A. 2006, MNRAS, 371, 19

Tsygankov, S. S., Lutovinov, A. A., Doroshenko, V., et al. 2016a, A\&A, 593, A16

Tsygankov, S. S., Mushtukov, A. A., Suleimanov, V. F., \& Poutanen, J. 2016b, MNRAS, 457, 1101

van Paradijs, J., Schlosser, W., Tarenghi, M., Sanduleak, N., \& Philip, A. G. D. 1977, IAU Circ., 3134

Walter, R., Lutovinov, A. A., Bozzo, E., \& Tsygankov, S. S. 2015, A\&ARv, 23, 2

Wang, Y.-M. 1987, A\&A, 183, 257

Weng, S.-S., Ge, M.-Y., Zhao, H.-H., et al. 2017, ApJ, 843, 69 